\title{
IDENTIFICACIÓN DE LAS ESPECIES IBÉRICAS Y BALEÁRICAS DEL GÉNERO POTAMOGETON L. EN ESTADO VEGETATIVO
}

\section{P. García Murillo}

Dpto. de Biología Vegetal y Ecología, Facultad de Farmacia, Universidad de Sevilla. ci Tramontana, sin., 41080 Sevilla. España.

Palabras clave: Potamogeton, Península Ibérica, taxonomía, anatomía, macrófitos.

\author{
ABSTRACT \\ IDENTIFICATION OF IBERIAN AND BALEARIC SPECIES \\ OF THE GENUS POTAMOGETON L. IN VEGETATIVE STATE
}

On the basis of vegetative, morphological and anatomical characteristics, a dichotomous key is provided for the identification of species and hybrids of the genus Potamogeton L. occurring in the Iberian Peninsula and Balearic Islands.

\section{INTRODUCCIÓN}

El género Potamogeton L. incluye cerca de 100 especies de distribución subcosmopolita (DAHLGKEEN et al., 1985). Se trata de uno de los géneros más notorios, en términos de biomasa y número de especies, entre los macrófitos sumergidos de nuestros ríos, arroyos y lagunas.

La identificación de sus especies se basa fundamentalmente en los caracteres de frutos, inflorescencias y hojas (HAYNES. 1978), y muchas veces reviste cierta dificultad, puesto que es frecuente encontrar poblaciones en estado vegetativo, ya sea por tratarse de poblaciones híbridas, porque la época no es la propicia para la fructificación, o bien, porque se encuentre ante una de las numerosas poblaciones que están durante anos sin fructificar.

El objeto de este trabajo es proporcionar unas claves de identificación fidedignas y de manejo relativamente fácil, que permitan la identificación de las distintas especies de Potamogeton que viven en la Península Ibérica e Islas Baleares según

Limnetica, 7: 71-82 (1991)

(C) Asociación Espanola de Limnología, Madrid. Spain los caracteres vegetativos y en especial los anatómicos, presentes en las poblaciones en todo momento.

\section{MATERIAL Y MÉTODOS}

El estudio morfológico se realizó a partir del examen de más de un millar de ejemplares recogidos por diversos recolectores y por el autor, por toda la zona de estudio, y que se encuentran depositados en 38 herbarios nacionales y extranjeros. Tanto las localidades como los herbarios están reseñados en García Murillo (1990).

El estudio de los caracteres anatómicos fue efectuado a partir de 133 muestras correspondientes a los 20 taxones reconocidos en la Península Ibérica y Baleares; ver Garcia Murillo (1990). El número de muestras estudiadas por taxon variaba entre 2 y 5 dependiendo de la distribución geográfica, complejidad taxonómica y variabilidad del taxon. Se utilizó fundamentalmente material seco, si bien en los casos en los que fue difícil su hidratación se utilizó material fresco conservado en formaldehído al $10 \%$. 
Tabla 1.- Synopsis del género Potamogeton L. en la Peninsula Ibérica y Baleares.

Synopsis of the genus Potamogeton L. in Iberian Penninsula and Balearic lslands.

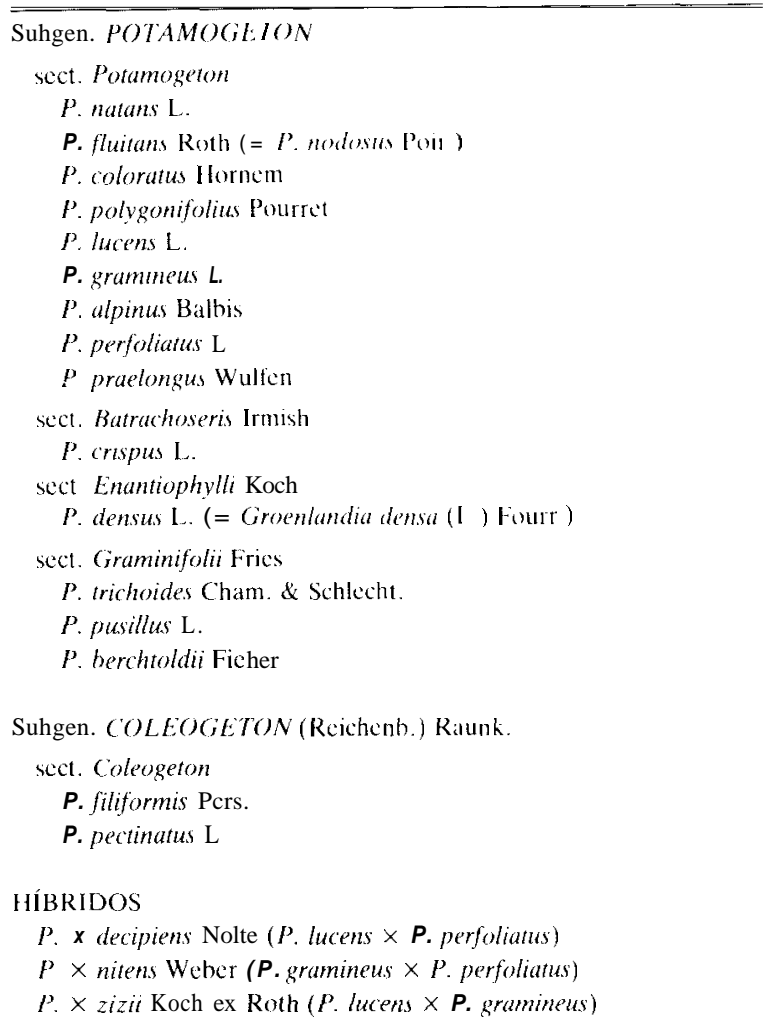

Los cortes se efectuaron a mano alzada con una cuchilla de afeitar y para su observación se siguieron los pasos que a continuación se indican: 1) se hidrataron las muestras sumergiéndolas en agua caliente durante algunos minutos; 2) se pasaron a hipoclorito de sodio $(40 \mathrm{~g} / \mathrm{l})$ durante 5 minutos con el fin de destruir todo el protoplasma y dejar sólo la pared celular; 3 ) se lavaron con agua durante 3 minutos; 4) se incluyeron en ácido láctico para su observación.

En las muestras en que los caracteres eran difíciles de observar o bien cuando se querían hacer más destacables se utilizó la tinción de paredes celulares diferencial Safranina-Verde Luz (RomeIs, 1928).

En la ordenación de los distintos taxones considerados en este trabajo se ha seguido la propuesta por García Murillo (1990).

\section{RESULTADOS}

En la Península Ibérica se conocen 20 taxones pertenecientes a los dos subgéneros en que se divide tradicionalmente el género Potumogeton (tabla 11 : subgen. Potamogeton y subgen. Coleogeton (Reichenb.) Raunk. Del subgen. Potamogeton. el más diverso y caracterizado por presentar las estípulas libres de las hojas, hallamos cuatro secciones: sect. Potamogeton, con hojas anchas, alternas y carpelos libres. con nueve especies (P. natans $\mathrm{L}$., P. fluitans Roth, P. colorutus Hornem.. $P$. polygonifolius Pourret, $P$. lucens L., $P$. gramineus L., P. alpinus Balbis, $P$. perfoliatus L. y $P$. praclongus Wulfen); sect. Batruchoseris Irmish, separable de la sección anterior por sus carpelos connados en la base, con una especie (P. crispus L.); scct. Enantiophylli Koch, con hojas anchas, subopuestas y carpelos libres, también con una especie (P. densus L.); y sect. Graminifolii Fries, que se distingue de las anteriores por sus hojas estrechamente lineares, con tres especies (P. trichoides Cham. \& Sclecht., P. pusillus L. y P. berchtoldii Fieber). El subgénero Coleogeton (Reichenb.) Raunk., incluye menor número de especies y su carácter principal consiste en que las estípulas están soldadas a las hojas formando una vaina que envuelve el tallo. Presenta sólo una sección con dos especies (P. filiformis Pers. y P. pectinatus L.). Además tres híbridos interespecíficos han sido localizados en la Península Ibérica: P. x decipiens Nolte (P. lucens L. $\times$ P. perfoliatus L.), $P . \times$ nitens Weber $(\mathrm{P}$. gramineus $\mathrm{L} . \times P$. perfoliatus L.) y $P . \times$ zizii Koch ex Roth (P. lucens L. $X$ P. gramineus L.).

\section{Caracteres utilizados}

a) Tallos

Los caracteres de mayor valor diagnóstico se han encontrado en las secciones de los entrenudos de los tallos y se observan en el córtex, endodermis y estela (fig. 1).

\section{Córtex}

Esta región del tallo, situada entre la epidermis y el cilindro central, está formada por células grandes, isodiamétricas y con paredes delgadas. Las células están dispuestas en varias filas y dejan 
entre ellas numerosos espacios esquizógenos, llamados lagunas aeríferas, las cuales se disponen radialmente en uno o más círculos, según las especies, decreciendo en su tamaño a medida que nos acercamos al cilindro central. Ver fig. 1, A y fig. 3 .

En algunas especies, entre las lagunas aeriferas y en las zonas límite entre la hipodermis y el córtex, aparecen haces fibrovasculares (fig. 1 A). Estos haces están formados por floema rodeado de varias fibras de esclerénquima, o bien, por un haz de fibras, por pérdida del floema (SHENK, 1886; Chrysler, 1907; Monoyer, 1927; Ogden, 1943; TOMLINSON, 1982; etc.).

Los caracteres más importantes que presenta el córtex son la presencia o ausencia de haces fibrovasculares y la forma en que se disponen. Según esto se pueden distinguir tres tipos de cortex: con haces fibrovasculares por todo él, con haces fibrovasculares en uno o dos anillos en la porción más externa y sin haces fibrovasculares. En la fig. 3 se pueden observar estos tres tipos: P. natans L. (A) y $\mathrm{P}$. pruelongus Wulfen $(\mathrm{H})$, representan el primer caso; P. lucens L. (D) y P. grumineus L. (E), el segundo y el tercero P. fluituns Roth (B), P. coloratus Hornem. (C), P. alpinus Balbis $(F)$, P. perfoliutus L. ( $G$ ) y P. crispus L. (I).

Las especies del subgen. Coleogeton (Reichenb.) Raunk. presentan unos pocos cordones, generalmente en número variable, que se disponen en un anillo en la zona central del cortex.

Tipo de células endodérmicas

En el material estudiado se discriminó entre dos tipos de células endodérmicas según los engrosamientos que presentan sus paredes celulares. Por una parte las células $\mathbf{O}$, con engrosamiento uniforme de las paredes celulares (P. fluituns Roth. fig. $3 \mathrm{~B}$ ) y, por otra, las células $\mathrm{U}$, con únicamente las paredes lateral e interna engrosadas. (P. natans L., fig. 1 A).

\section{Estela}

El cilindro vascular presenta una sección transversal oblonga, elíptica o casi circular y está constituida por un tejido medular donde se hallan incluidos los haces vasculares (fig. 1); cada haz suele estar rodeado, al menos en parte, de una vaina de esclerénquima (TomLinson, 1982). El floema está formado por grandes células poligonales, con las paredes delgadas, de manera que, a veces, es difícil distinguirlo del resto de las células medulares. El xilema desaparece de esta zona del tallo originando una laguna xilemática, al igual que ocurre en otras hidrofitas (SHENk, 1886; ARBER, 1920; S( uithorpe, 1968), encontrándose sólo en las regiones nodales, donde se hallan traqueidas con engrosamientos anulares o espiralados (CirysilR, 1907; Monoyer, 1927).

En un corte transversal se pueden distinguir en la estela una región central y dos laterales. La región central determina el eje que pasa por los vasos de mayor desarrollo, los cuales pueden ser: colaterales, bicolaterales o concéntricos. Las regiones laterales pueden llevar o no vasos, los cuales son siempre colaterales.

Dependiendo del número de vasos presentes y su disposición, se pueden hacer una serie de modelos estelares básicos que son propios de distintos grupos de especies. En base a la clasificación que hicieron Raunkiaer (1903), Chrysler (1907), Hagström (1916) y OGden (1943) se describen los siguientes modelos básicos:

- Tipo Proto: En esta estela (fig. 1 B) se suelen encontrar 10 haces vasculares, siendo éstos independientes unos de otros, y todos colaterales. En la región central se disponen enfrentados dos grandes y junto a ellos dos más pequeños, formando todos una especie de cruz. El resto de los haces se disponen en las regiones laterales. En la fig. 3 se observan en P. coloratus Hornem. (C) y $\mathrm{P}$. praelongus Wulfen $(\mathrm{H})$.

- Tipo Trio (sensu Chrysler, 1907). Tres de los haces de la región central (uno grande y dos pequeños) que presentaba la estela de tipo Proto se han unido formando un solo haz (fig. 1 A y C). Este haz está compuesto por una laguna central de origen xilemático y por tres partes de floema, dos hacia un lado de la laguna y otra en el lado opuesto (a veces el haz tipo Trio queda reducido a un haz colateral, como ocurre en algunos ejemplares de P. fluituns Roth). El haz tipo Trio se dispone frente al otro haz de la región central, que al igual que el resto de los haces vasculares continúan siendo colaterales. Así resultan un haz tipo Trio y otro colateral en la región central y varios, generalmente tres en cada lado, en las regiones laterales, como puede verse en la fig. 3 en P. natuns L. (A), P. fluituns Roth (B), P. alpinus Balbis $(F)$ y $P$. perfoliatus $L .(G)$. 
- Tipo Oblongo: En esta estela las tres regiones están separadas. generalmente, por un conjunto de células de esclerénquima. En el caso más simple se encuentra un haz colateral en cada una de las regiones laterales y un único haz bicolateral en la región central (fig. $1 \mathrm{E} ; P$. gramineus $\mathbf{L}$. fig. $3 \mathrm{E}$ y $P$. crispus L. fig. 3 1). Algunas poblaciones de P. lucens L. presentan 2(-3) haces en las regiones laterales, aunque, en la región central es posible hallar dos haces colaterales muy próximos (fig. 1 D).

- Tipo Simple: Es el resultado de la fusión dc los haces del modelo de estela Oblongo, que se unen en un sólo haz concéntrico anficribal (fig. 1F). Esto ocurre en las especies de la sect. Graminifolii Fries y del subgen. Coleogeton (Reichenb.) Raunk. En P. filiformis Pers. se pueden encontrar 2 lagunas xilemáticas muy juntas y de igual tamaño rodeadas de floema, o incluso 4 lagunas xilemáticas de iguales características.

\section{b) Hojas}

En el género Potamogeton L. la disposición de las hojas es alterna, si bien en P. densus L., las hojas se disponen de forma subopuesta. La forma, tamaño y consistencia de las hojas son muy variables dentro del género y, en general, aportan numerosos caracteres útiles para la separación de los distintos taxones (fig. 2).

Pueden ser de formas anchas (sect. Potamogeton, sect. Batruchoseris Irmish y sect. Enantiophylli Koch) o lineares [sect. Gruminifolii Fries y subgen. Coleogeton (Reichenb.) Raunk.]. Las primeras pueden ser sésiles o pecioladas, con ápice agudo u obtuso, margen entero, finamente denticulado o serrulado, y base atenuada, obtusa o amplexicaule. En las segundas, un carácter importante, al igual que ocurre en géneros próximos, Ruppia L. y Zannichellia L. (LuTHER, 1947; TA. LAVERA et al., 1986), es la forma del ápice, que puede ser agudo, como en P. trichoides Cham. \& Schlecht. (fig. $2 \mathrm{H}$ ) y P. pectinutus L. (fig. $2 \mathrm{E}$ ), obtuso, como en P. filiformis Pers. (fig. 2C), y obtuso mucronado, como en P. pusillus L. (fig. 21), $P$. berchtoldii Fieber (fig. $2 \mathrm{~J}$ ) y P. pectinutus $L$. (fig. $2 \mathrm{~J}$ ).

La consistencia del limbo, en las hojas de formas anchas, puede ser coriácea o membranácea, según dos tipos de organización anatómica del limbo foliar (García Murillo, 1990). Cuando el limbo es opaco se corresponde con la textura coriácea, propia de las hojas flotantes. El limbo translúcido se corresponde con la consistencia membranácea y lo presentan las hojas sumergidas dc todas las especies de hojas anchas.

\section{c) Estípulas}

Están presentes en todas las especies de Potamogeton, aunque en $P$. densus L. sólo se encuentran en las brácteas. Se pueden encontrar las estípulas soldadas a las hojas como se observa en P. filiformis Pers. y P. pectinutus L. (fig. 2 A), o bien libres (fig. 2 B, F y G) como en el resto de las especies ibéricas. Entre estas últimas, en los taxones con hojas lineares, es posible discriminar entre especies con estípulas convolutas (HAGSTRÖM, 1916), cuando están abiertas en toda su longitud, aunque aparentemente están cerradas, como ocurre en P. trichoides Cham. \& Schlecht. y P. herchtoldii Fieber (ver fig. 2 F), y especies con estípulas connadas en su base (HAGSTRÖM, 1916), cuando forman un tubo en su tercio inferior, como se observa en P. pusillus L. (ver fig. $2 \mathrm{G}$ ). Estos caracteres se deben de observar en ramas jóvenes.

Respecto a su consistencia, pueden ser membranosas (como en $P$. fluitans Roth), herbáceas (como en P. lucens L.) y fibrosas (como en P. $n a-$ tans L.), siendo las primeras caducas (no se encuentran enteras en los entrenudos inferiores) y las dos últimas persistentes (se encuentran completas en todos los entrenudos).

\section{Clave}

1. Estípulas soldadas a las hojas formando una vaina que abraza al tallo ......................... 2 - Estípulas no soldadas con las hojas, formando una vaina intrafoliar que abraza el tallo, o sin estipulas ....................................... 3

2. Ápices de las hojas obtusos, sin mucrón. Cilindro central del tallo con dos o cuatro lagunas xilemáticas muy próximas entre sí, rodeadas de floema (estela tipo Simple) ......... P. filiformis Pers. - Ápices de las hojas agudos u obtusos, mucronados. Cilindro central del tallo con una única laguna xilemática rodeada de floema (estela tipo Simple) ....................... P. pectinatus L. 

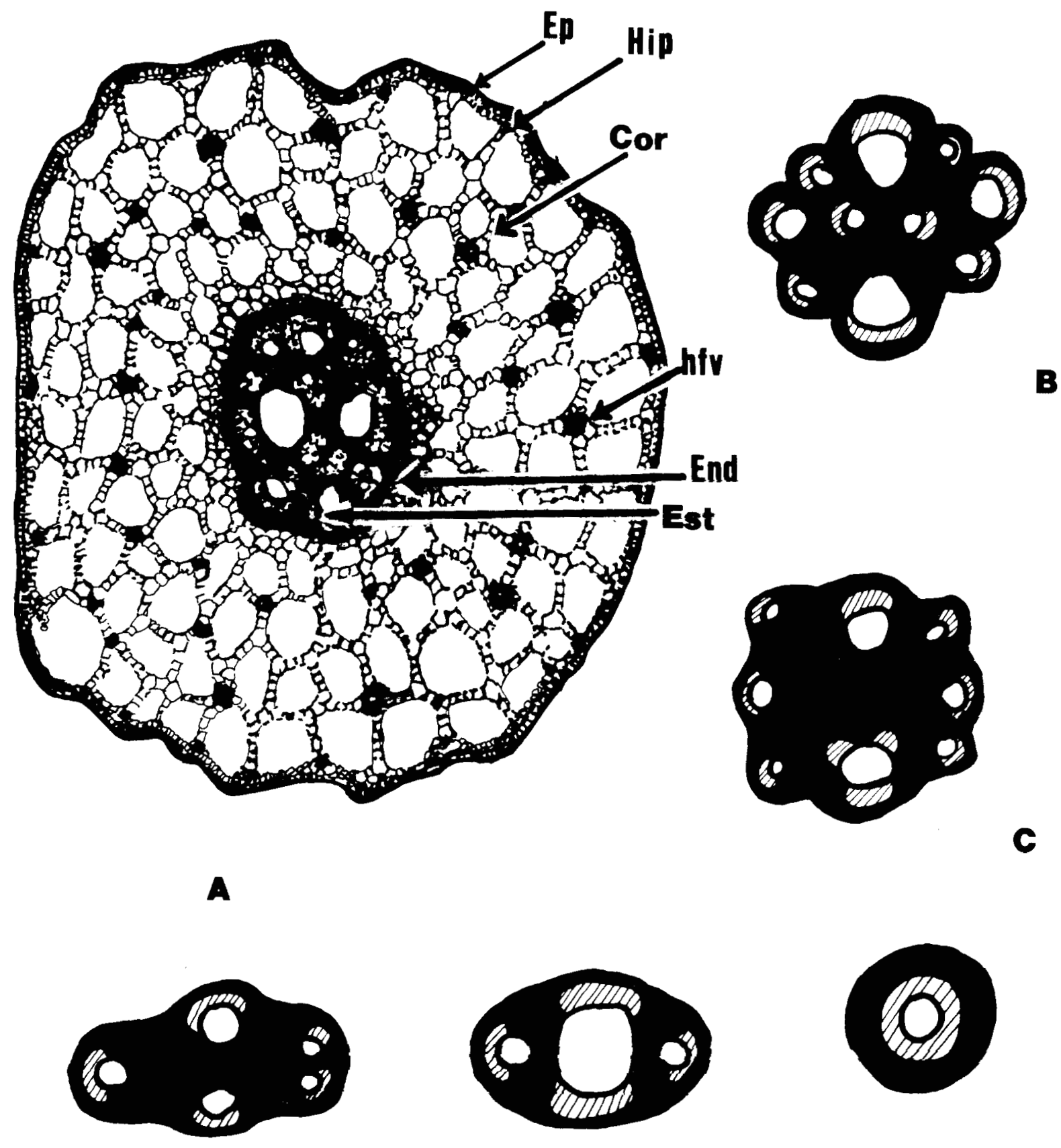

D
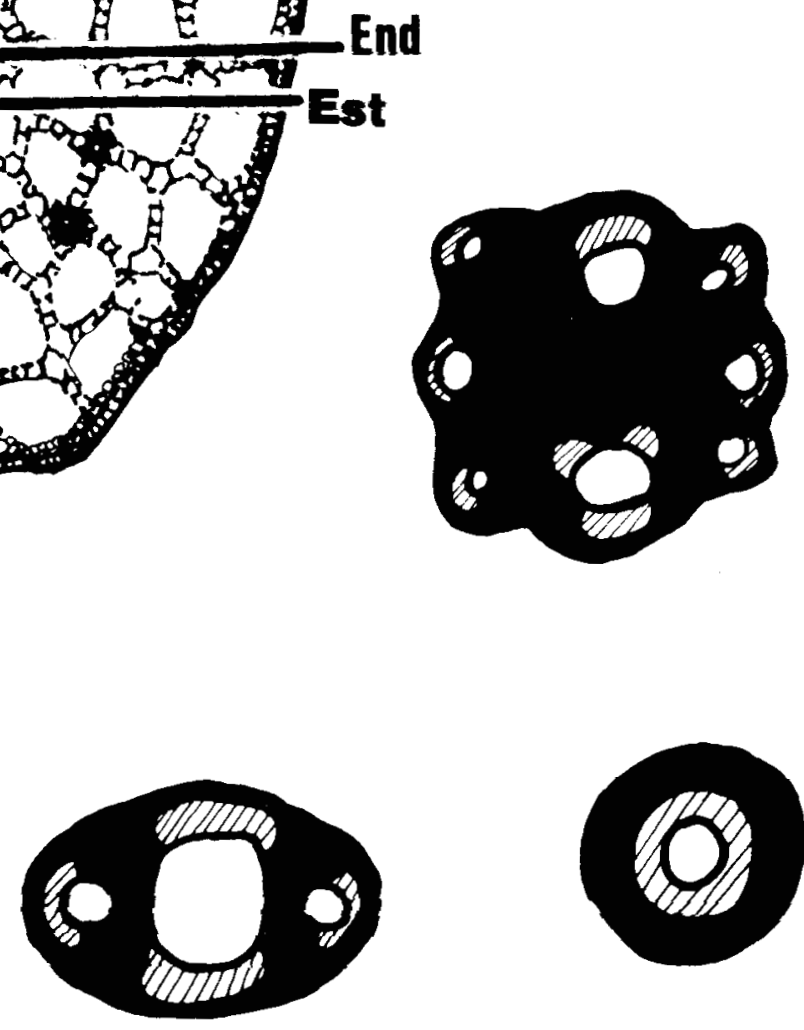

C

$\mathbf{B}$

\section{C} fibrovascularcs. End. endodermis (de tipo U). Est. estela. Tipos de estela, X50: b) tipo Proto (P. coloratus): c) tipo Trío (P. fluitans); d) tipo Oblongo (P. lucens); e) tipo Oblongo (P. gramineus) y f) tipo Simple (P. trichoides).

a) Cross section of stem of P. natans (Cáceres, Casatejada). Ep. epidermis. Hip. hypodermis. Cor. cortex. hfv. fibrovascular bundles. End, endodermis (U-type). Est. stela.

Types of stele, X50: b) Proto type (P. coloratus); c) Trío type (P. fluitans); d) Oblong type (P. lucens); e) Oblong type (P. gramineus) and f) Simple type (P. trichoides). 
3. Todas las hojas del tallo estrechamente lineares. las más anchas de menos de $3 \mathrm{~mm}$. Tallos con estela de un único haz concéntrico anficribal (tipo Simple) - Al menos las hojas más superiores de lanceoladas a ovadas, más anchas de $3 \mathrm{~mm}$. Tallos con estelas de. al menos, tres haces vasculares, colaterales, bicolaterales, o con una laguna xilemática y tres partes de floema (tipos Oblongo, Trio y Proto)

4. Ápices de las hojas agudos. Anchura de las hojas generalmente menor de $0,7 \mathrm{~mm}$. Nervio medio de las hojas sobresaliendo a modo de costilla, especialmente en la base, sin lagunas aeríferas junto al nervio central, nervios laterales muy poco evidentes. Tallo del mismo color, verde oscuro, que las hojas

P. trichoides Cham. \& Schlecht.

- Ápices de las hojas obtusos y mucronados. Anchura de las hojas por lo general mayor de $1 \mathrm{~mm}$. Nervio medio de las hojas apenas sobresaliendo del resto del limbo, generalmente con una o dos filas de lagunas aeríferas junto al nervio central, en algunas ocasiones nervios laterales apreciables. Tallos de color amarillento o del mismo color que las hojas 5

5. Estípulas de las hojas connadas. P pusillus L. - Estípulas de las hojas convolutas P. berchtoldii Fieber

6. Hojas subopuestas, sin estípulas. P. densus L.

- Hojas alternas, todas estipuladas ............ 7

7. Hojas amplexicaules o semiamplexicaules, al menos las basales ................................... 8 - Hojas sésiles o pecioladas, no amplexicaules 12

8. Estípulas persistentes. Cortex con haces fibrovasculares. Endodermis de tipo U
- Estípulas caducas. Cortex sin haces fibrovasculares. Endodermis de tipo $\mathrm{O}$.................. 9

9. Hojas ovales o subrotundas, ampliamente amplexicaules. Margen finamente denticulado. Limbo con 5 nervios o más. Estela con 8 haces vasculares P. perfoliatus L. - Hojas oblongas o estrechamente oblongas, débilmente amplexicaules. Margen, generalmente, serrulado. Limbo con 3 nervios. Estela con 3 haces vasculares alineados P. crispus L.

10. Estípulas fibrosas, blanquecinas. Estela con, al menos, 10 haces vasculares (tipo Proto). Hojas con margen entero .......... P. praelongus Wulff. - Estípulas herbáceas, verdosas o parduscas. Estela con 5 (tipo Oblongo)-8 (tipo Trio) haces vasculares. Hojas con margen finamente denticulado (ver en los ápices de las hojas jóvenes) ........ 11

11. Hojas elípticas con ápice cuspidado. Estela con 5 (tipo Oblongo)-8 (tipo Trio) haces vasculares. Endodermis O ó U

P. $\times$ decipiens Nolte (P. lucens $\times$ P. perfoliatus) - Hojas oblanceoladas, con ápice agudo. Estela con 5 haces vasculares (tipo Oblongo). Endodermis tipo U

P. $\times$ nitens Weber (P. gramineus X P. perfoliatus)

12. Algunas hojas claramente pecioladas (pecíolo $>10 \mathrm{~mm}$ ) .................................... 13

- Todas las hojas sentadas o con pecíolos muy

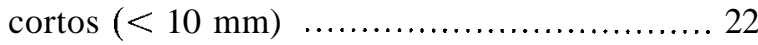

13. Cortex cori haces fibrovasculares $\ldots \ldots \ldots \ldots . .14$

- Cortex sin haces fibrovasculares ............. 19

14. Estela con 10 haces vasculares (tipo Proto). Endodermis de tipo O. Estípulas caducas, con frecuencia casi negras. Tallos generalmente con nudos enraizantes y entrenudos muy cortos $(<15 \mathrm{~mm})$............. P. polygonifolius Pourret

Figura 2.- a) P. fluitans (Sevilla, Cazalla de la Sierra), X1/2. Hábito hf. hojas flotantes. hs, hojas sumergidas. Es, estipulas. b) Base de la hoja de P. pectinatus, X5: v, vaina. li. ligula. Apices de hojas. X5: c) P. filiformis, d) y e) P. pectinutus, h) P. trichoides, i) P. pusillus, j) P. berchtoldii. Estipulas intrafoliares, X5: f) convolutas (P. berchtoldii); g) connadas en la base (P. pusillus).

a) P. fluitans (Sevilla. Cazalla dc la Sierra), X1/2. Habit. hf, floating leaves. hs, submerged leaves. Es, stipules. b) leaf basis of P. pectinatus, X5: v. leaf sheaths, li, ligule. Leaf tips, X5: c) P. filiformis. d) and e) P. pectinatus. h) P. trichoides. i) P. pusillus. j) P. berchtoldii. Intrafoliar stipules, X5: f) convolute (P. berchtoldii); g) connate in the lower part (P. pusillus). 


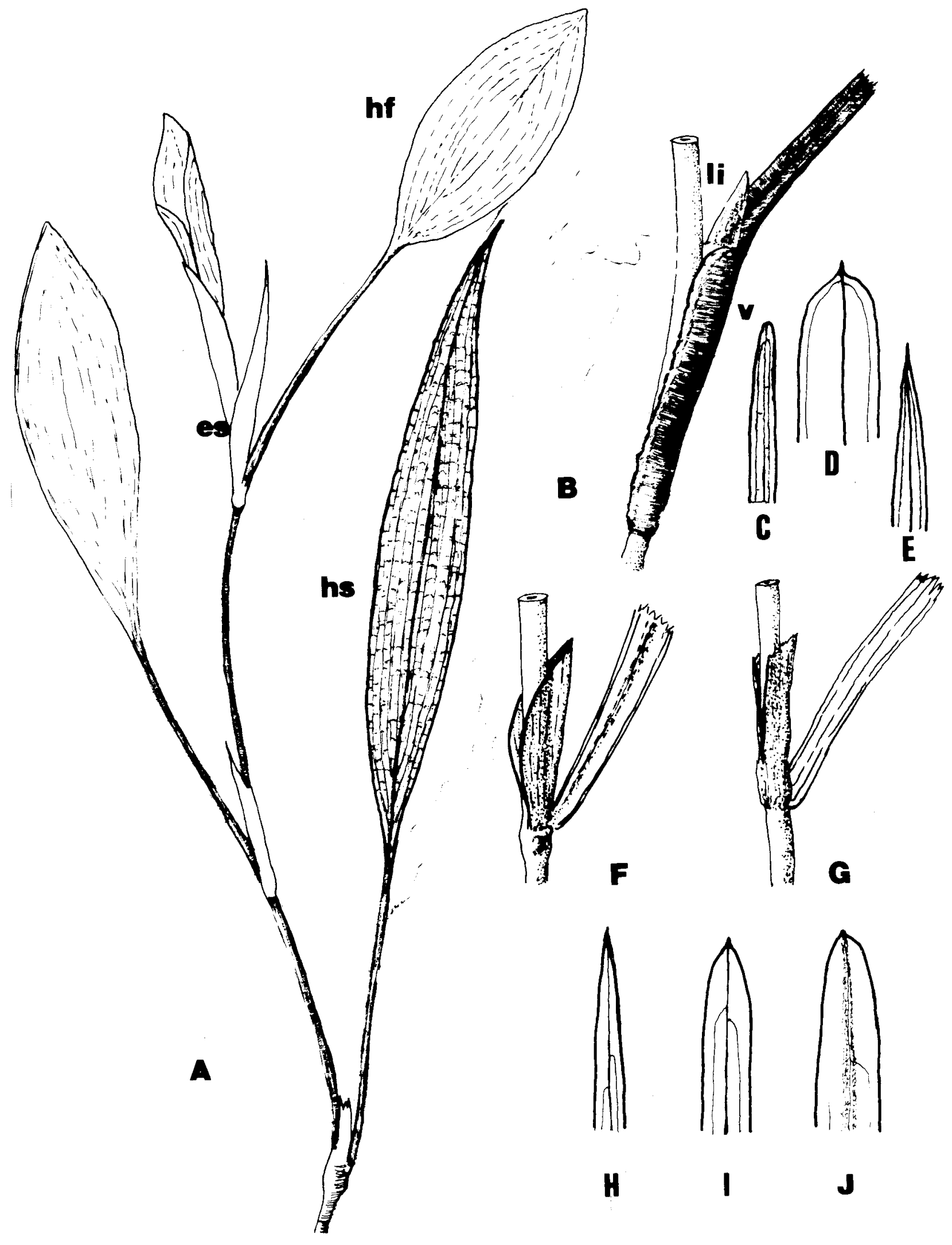


- Estela con 8 (tipo Trio) o menos (tipo Oblongo) haces vasculares. Endodermis de tipo U. Estípulas persistentes, blanquecinas, verdes o parduscas. La mayoría de los nudos no enraizantes y entrenudos largos (>20 mm) 15

15. Todas las hojas membranáceas (translúcidas), base atenuada, ápice cuspidado 16 - Todas las hojas coriáceas (opacas), o sólo las más superiores, base cordada o atenuada, ápice

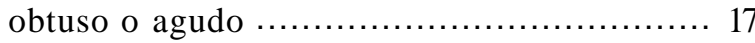

16. Todos los pecíolos de igual tamaño y muy cortos. Hojas todas semejantes ......... P. lucens $L$. - Pecíolos de las hojas superiores y algunas del tallo principal mayores que los de las inferiores y ramas laterales, que prácticamente no lo presentan. Hojas superiores más estrechas y cuspidadas que las inferiores o las de las ramas laterales ..... $\mathrm{P}$. $\times$ zizii Koch ex Roth $(\mathrm{P}$. lucens $\times \mathrm{P}$. gramineus)

17. Estela con 8 haces vasculares (tipo Trio). Estípulas fibrosas generalmente blanquecinas. Todas las hojas coriáceas, o bien las inferiores transformadas en filodios. Haces fibrovasculares distribuidos por todo el cortex .............. P. natans L. - Estela con 3-5 haces vasculares (tipo Oblongo). Estípulas herbáceas, verdosas o parduscas. Sólo las hojas más superiores coriáceas, el resto membranáceas. Haces fibrovasculares en los 2-3 anillos periféricos o por todo el cortex 18

18. Hojas pecioladas sólo en los últimos nudos, ovadas o elípticas, obtusas, de 20-67 × 8-45 mm. Tallos muy ramificados. Haces fibrovasculares en la periferia del cortex. Estela con tres haces vasculares alineados (tipo Oblongo)

P. gramineus $\mathrm{L}$. - Hojas pecioladas a lo largo del tallo principal, oblanceoladas o estrechamente elípticas, cuspidadas, de 45-110 × 7-30 mm. Tallos poco ramifica- dos. Haces fibrovasculares por todo el cortex. Estela con 5 haces vasculares (tipo Oblongo) ......... P. $\times$ zizii Koch ex Roth $(\mathrm{P}$. grurnineus $\times$ P. lucens)

19. Todas las hojas membranáceas (translúcidos) ......................................... 20 - Hojas, al menos las más superiores, coriáceas (opacas) 21

20. Estela con 8 haces vasculares (tipo Trio). Ápiccs foliares agudos. Hojas todas oblanceoladas, agudas, con margen finamente denticulado (ver en ápices de hojas jóvenes) y de 95-210 × 10-20 mm.

\section{P. fluitans Roth}

- Estelas con 10 haces vasculares (tipo Proto). Ápices foliares obtusos. Hojas inferiores oblanceoladas, las superiores ovadas, ambas obtusas, con margen entero y de 35-200 $\times 17-47 \mathrm{~mm} \ldots$...

P. coloratus Hornem.

21. Hojas superiores coriáceas espatuladas con un corto pecíolo $(<10 \mathrm{~mm})$. Hojas medias y basales membranáceas, obtusas y sentadas

P. alpinus Balbis

- Hojas superiores coriáceas oblanceoladas o elípticas, con un pecíolo de 20-252 mm. Hojas medias y basales membranáceas agudas y la mayoría pecioladas ...................... P. fluitans Roth

22. Estípulas persistentes. Ápice agudo o cuspidado. Haces fibrovasculares en el cortex. Endodermis de tipo U. Estela 3-5 haces vasculares .... 23

- Estípulas caducas. Ápice obtuso. Sin haces fibrovasculares en el cortex. Endodermis de tipo O. Estela con 8 (tipo Trio)-3 (tipo Oblongo) haces vasculares 24

23. Hojas elípticas y obtusas, cuspidadas u oblanceoladas y agudas, con base atenuada en un corto pecíolo, anchura de las hojas, generalmente, ma-

Figura 3.- Secciones de tallos: a) P. natans (Alava, Tuy); b) P. fluitans (Sevilla, El Real de la Jara); c) P. coloratus (Murcia, Moratalla); d) P. lucens (Huelva. Doñana, El Sopetón); e) P. gramineus (Guadalajara, Tortuera); f) P. alpinus (Huesca, Ibón de Anayet); g) P. perfoliatus (Lugo, Palas de Rey); h) P. praelongus (Lérida, Balsa del Xic); i) P. crispus (Valencia, Silla).

Cross section of stems: a) P. natans (Alava, Tuy); b) P. fluitans (Sevilla, El Real de la Jara); c) P. coloratus (Murcia, Moratalla): d) P. lucens (Huelva, Doñana, El Sopetón); e) P. gramineus (Guadalajara, Tortuera); f) P. alpinus (Huesca, Ibón de Anayet); g) P. perfoliatus (Lugo, Palas de Rey); h) P. praelongus (Lérida, Balsa del Xic); i) P. crispus (Valencia, Silla). 


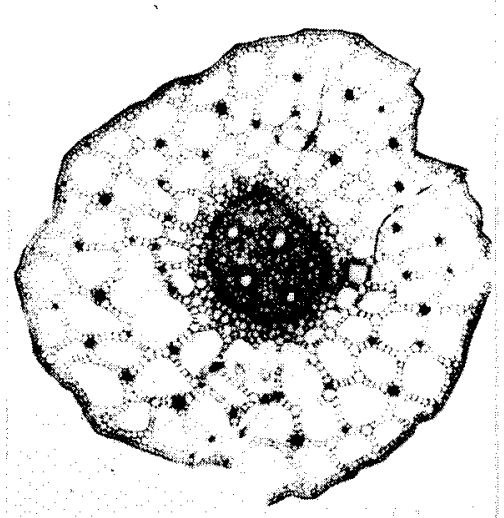

A

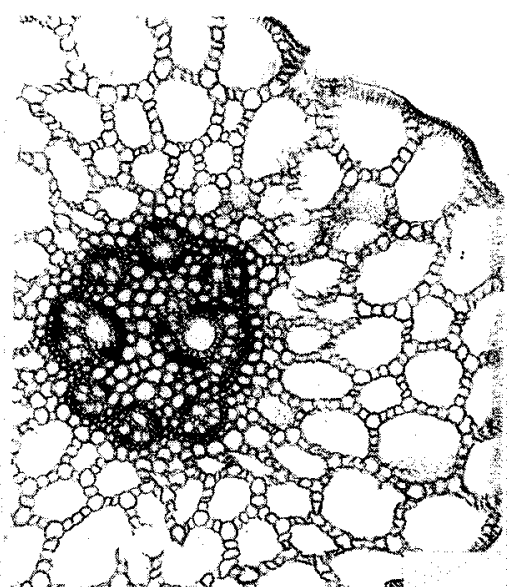

poratsons

B
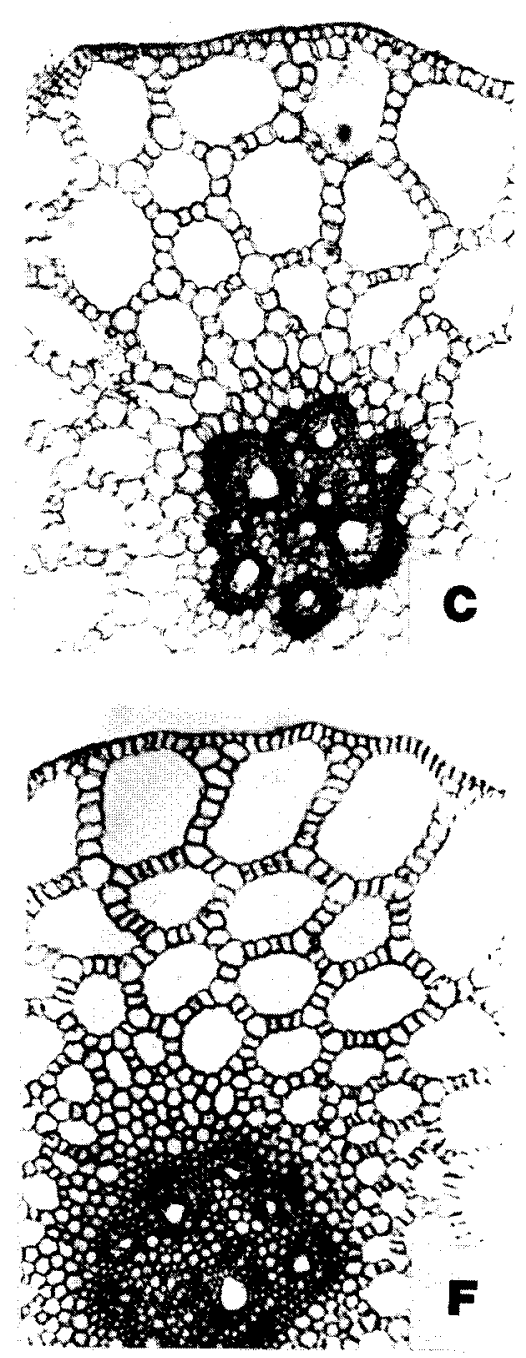

E
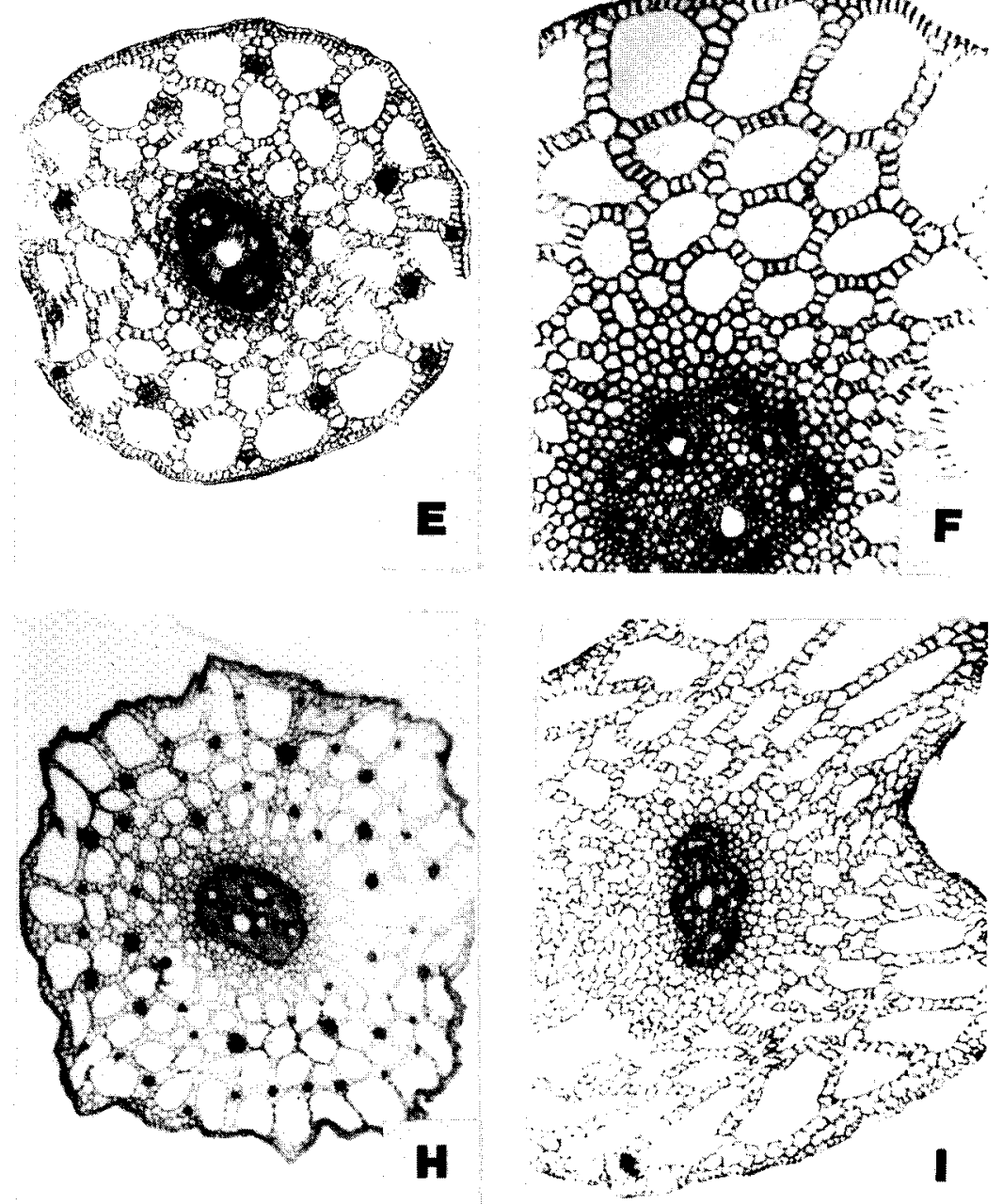
yor de $15 \mathrm{~mm}$. Estela con 3, 4 ó 5 haces vascula-

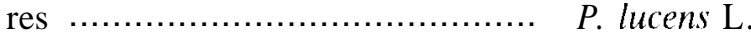
- Hojas lanceoladas o anchamente lineares y agudas, sentadas, anchura de las hojas, generalmente, menor de $15 \mathrm{~mm}$. Estela con sólo tres haces vasculares alineados $\ldots \ldots \ldots . . P$. gramineus $L$.

24. Estela con 3 haces vasculares generalmente alineados (tipo Oblongo). Hojas oblongas o anchamente oblongas, margen con frecuencia serrulado, con tres nervios ,.............. P. crispus $L$. - Estela con 8 haces vasculares (tipo Trio). Hojas oblanceoladas, margen entero, con 5 ó más nervios P. alpinus Balbis

\section{Variabilidad}

Se han detectado algunas poblaciones donde los individuos morfológicamente corresponden a $P$. natans, si bien, todas sus estructuras presentan un tamaño mucho más pequeño. Por otra parte, los caracteres anatómicos del tallo muestran una variabilidad atípica en el resto de las poblaciones de la especie. Así, se ha encontrado un ejemplar con una endodermis de tipo $\mathrm{O}$, siendo lo típico las de tipo U. Las estelas que presentan estas poblaciones varían entre el tipo Trio y el tipo Oblongo, con dos haces vasculares en la región central y uno en cada región lateral, existiendo una variabilidad continua entre uno y otro tipo de estela. A estas poblaciones se las ha identificado como $P$. natans var. variifolus (Thore) Roemer \& Shultes (GARCia Murillo, 1990).

En una población de los Pirineos (Ibón de Sabocos, Huesca) se han encontrado ejemplares que morfológicamente se identifican con $P$. gramineus L., cuya estela presenta 5 haces vasculares en lugar de 3, como es lo normal en los ejemplares de esta especie (Hagstrom, 1916). Esto podría apoyar la idea de que dicha población sea de origen hibridógeno.

Una de las poblaciones estudiadas de $P$. alpinus (Ibón de Piedrafita, Huesca) presenta ejemplares con estela de tipo Proto, aunque lo usual en la especie es el tipo Trio. La misma anomalía ha sido estudiada por el autor en otras poblaciones europeas (Luxemburgo, Ardenas), coincidiendo con lo descrito anteriormente por RAUNKIAER (1903), HAGSTRÖM (1916), MONOYER (1927) y OGden (1943).

\section{Notas sobre la ecología}

La practica totalidad de las especies de Potamogeton se encuentran en aguas continentales y permanentes. mostrando mayor preferencia por las aguas lentas que por las aguas de curso rápido.

Respecto a los factores químicos, la mayoría de las especies dc la Península Ibérica viven en aguas dulces. $P$. pectinatus L. es la especie más eurioica, admitiendo niveles de eutrofia mucho mayores que las otras y viviendo en aguas que presentan el mayor rango de concentración iónica entre todas las especies de Potamogeton (PIP, 1987), se halla con frecuencia tanto en aguas salinas como en salobres. P. fluitans Roth, P. crispus L. y P. pusillus L. muestran también una gran tolerancia a valores altos de alcalinidad (FERRER \& COMÍN, 1979; Hellouist, 1980; Margalef Mir, 1981; KADONO, 1982). P. coloratus Hornem. y P. densus $L$. son propios de aguas limpias, oxigenadas y ricas en Ca" (Cirujano, 1990). P. berchtoldii Fieber se encuentra tanto en aguas con valores moderados (Hellouist, 1980) como bajos (Margalef Mir, $1981)$ de reserva alcalina. $P$. polygonifolius Pourret es propio de aguas oligotrofas (HASLAM et al., 1975; ArnaIz \& Molina, 1985) o distrofas (Molina et al., 1988), nosotros lo hemos hallado siempre en lugares turbosos con aguas muy ácidas. P. natans L. es propio de aguas de baja alcalinidad (Margalef Mir, 1981; Kadono, 1982), oligomesotrofas (Molina et al., 1988), al igual que P. gramineus L. (Kadono, 1982; Arnaiz \& MoliNA, 1985). En aguas ácidas oligotrofas también hemos encontrado un gran número de poblaciones de P. trichoides Cham. \& Schlecht., lo mismo que Wiegleb et al. (1989), en cambio Margalef Mir (1981) y VeLAYos et al. (1984) señalan su presencia en aguas mineralizadas. Algo semejante parece ocurrir con P. perfoliatus L. (Hellouist, 1980; Kadono, 1982; Wiegleb et al., 1989). De P. lucens $L$. hemos localizado varias poblaciones en lagunas oligotrofas de aguas ácidas (P.N. Doñana: laguna del Sopetón, laguna del Taraje y laguna del Zahillo; ver parámetros químicos en BERnUES, 1990), si bien también tenemos noticias de su presencia en aguas básicas carbonatadas (Huesca, Pantano de Argués; Gerona, Lago de Bañolas). Finalmente P. alpinus Balbis, P. praelongus Wulfen y P. filiformis Pers. sólo han podido observarse en lagos glaciares Pirenaicos, la primera es- 
pecie es propia de aguas con alcalinidad baja (Margalef Mik, 1981; Pip, 1987), la segunda se halla dentro de un rango grande de valores de alcalinidad (Hellouist, 1980; KADONO, 1982) y la tercera es propia de aguas carbonatadas (PIP, 1987).

\section{DISCUSIÓN}

Tres factores principales dificultan la identificación de las especies del género Potamogeton L.: la escasez de estructuras, la variabilidad de sus caracteres y la presencia de híbridos interespecíficos.

La escasez de caracteres, más acentuada lógicamente en los órganos vegetativos, es consecuencia de la pérdida de estructuras que facilitan la vida en el medio terrestre, cutículas gruesas, estomas, indumentos, tejidos vasculares, etc. (DIEZ et al., 1988). En las especies estudiadas en este trabajo se pueden encontrar numerosos ejemplos de este proceso. Así, P. natans L., especie con hojas flotantes y con inflorescencias que se desarrollan fuera del agua, posee hojas con limbo y pecíolo, estomas, numerosos nervios (ca. de 13) y parénquima diferenciado en dos partes: parénquima clorofílico y parénquima lagunar, como cualquier planta terrestre. Lo mismo ocurre con P. fluitans Roth o con cualquier especie de hojas flotantes. En cambio, P. pectinatus L., especie con hojas totalmente sumergidas y con sus flores en el agua, posee hojas sin pecíolo, muy estrechas, sin estomas, con tres nervios y células del parénquima semejantes; se trata de una planta con estructuras más sencillas y mucho más adaptada a la vida en el agua. Igualmente puede verse la simplificación de los haces vasculares en la estela, desde especies con cerca de 10 haces vasculares, anfibias, como P. polygonifolius Pourret o P. natans L., hasta especies totalmente sumergidas con un único haz vascular, como $P$. pusillus L. o P. pectinatus L. (fig. 2).

A la complicación derivada de la escasez de estructuras hay que añadir una gran variabilidad en todos sus caracteres en sus dos componentes: de origen genotípico y fenotípico W IEGLEB (1988). En el primer caso debido al aislamiento de las poblaciones, como ocurre en las que viven en lagos glaciares o en lagunas, mantenidas por una fuerte multiplicación vcgetativa, y en el segundo por la acción del medio, siendo frecuentes especies con una especial sensibilidad a ciertos parámetros fisicoquímicos que se traducen cn variación de algunos de sus caracteres morfológicos. Esto último ocurre, por ejemplo, en $\mathrm{P}$. pectinatus L. donde la anchura y número de nervios de las hojas varían con respecto a la salinidad y los nutrientes del agua (V AN WiJk, 1988) o en $P$. perfoliatus L. con la forma y tamaño de las hojas con respecto a los iones del agua (PFarsal.l \& Hamby, 1925). Lo mismo ocurre en géneros cercanos como Ruppia (Richardoson, 1980). Por ello el problema fundamental reside en la separación entre la variación genotípica y la fenotípica, que, si bien, teóricamente son distinguibles, en la práctica es bastante más complejo y tediosos (WIEGLEB, 1988).

La aparición de híbridos interespecíficos en este género es muy frecuente y han sido descritos gran número de ellos (HaGström, 1916; OGden, 1943; DANDY, 1975; Haynes, 1978; etc.). Sin embargo, WIEGLEB (1988) reduce este número a una treintena, e insiste en que sólo pocos casos ocurren realmente con frecuencia y su distribución es a menudo muy localizada. Nuestras observaciones corroboran esta hipótesis, habiéndose encontrado híbridos solamente entre algunas especies anemófilas. Las poblaciones eran todas estériles y se localizaban siempre en las proximidades de alguno de los parentales. Sus caracteres tanto morfológicos como anatómicos, se incluyen dentro de la variabilidad mostrada por los parentales correspondientes, por lo que se hace necesario un buen conocimiento de las distintas especies para su correcta identificación.

La presencia de los tres factores anteriormente citados, se traduce en lo que WIEGLeB (1988) ha denominado una «situación nomenclatural desesperada». En este género los taxones se han descrito muchas veces basándose en un único ejemplar distinto de la descripción original, en plantas que viven en condiciones extremas, o han sido redescritos por varios autores. Al examinar numerosos pliegos y efectuar observaciones periódicas de campo, se percibe que tales variaciones suelen corresponder a estados de desarrollo y condiciones ambientales. Las malas determinaciones o los táxones superfluos son muchas veces transmitidos a la bibliografía, lo que amplifica de forma notable la confusión. Tales errores se podrían evitar o 
reparar efectuando un cuidadoso examen de los especímenes, contrastando las referencias bibliográficas, observando las plantas en su medio y comparando los ejemplares en estudio con los de otras colecciones.

\section{AGRADECIMIENTOS}

Agradezco a la Dra. M.J. Gallego su ayuda prestada durante la realización de este trabajo. También agradezco a los Dres. S. Talavera y J. Herrera sus comentarios acerca del mismo. Igualmente doy las gracias al Dr. A. Aparicio y a M. Arista por haber empleado su tiempo en probar las claves que se incluyen en este trabajo.

\section{Bibliografía}

ARBer, A., 1920. Water plants. A study of aquatic angiosperms. Cambridge University Press. London. 436 pp.

Arnaiz, C. \& J.A. Molina, 1985. Vegetación acuática y helofítica de la cuenca alta del río Guadarrama (Madrid, España). Lazaroa, 8: 221-240.

Bernues, M., 1990. Limnología de los sistemas acuáticos del Parque Nacional de Dotiana. Tesis Doctoral, Madrid. 242 PP.

Chrysler, M.A., 1907. The structure and relationship of the Potamogetonaceae and allied Families. Bol. Gaz., 44 (3): $161-188$

Cirujano, S., 1990. Flora y vegetación de las lagunas y humedales de la provincia de Albacete. Inst. Estudios Albacetenses (ser. I), 52. Albacete. 144 pp.

Dahlgreen, R.M.T., H.T. Clifford \& P.F. Yeo, 1985. The Families of the Monocotyledons. Berlin. 520 pp.

Dandy, J.E., 1975. Potamogeton. In: C.A. STACe (Ed.), Hybridization and Flora of the British Isles: 444-458. Academic Press. London.

Diez, M.J., S. Talavera \& P. Garcia Murillo, 1988. Contributions to the palynology of hydrophytic, non-entomophilous angiosperms. 1. Studies with LM and SEM. Candollea, 43: 147-158.

Ferrer, X. \& F. Comín, 1979. Distribució i ecologia dels macròfits submergits del Delta de l'Ebre. Butll. Inst. Cat. Hist. Nat., 44 (Sec. Bot. 3): 111-117

García Murillo, P., 1990. El género Potamogeton L. en la Península Ibérica. Universidud de Sevilla. Tesis Doctorales en microfichas, 4. Sevilla. $321 \mathrm{pp}$.

Hagstкom, J.O., 1916. Critical researches on Potamogetons. Kongl. Svenska Vetenskapasakad. Handl., 55: 1-281.
Haslam, S.M.. C.A. Sinker \& P.A. Wolseiey, 1975. British water plants. Fld. Stud., 4: 243-351.

Harnks, R.R., 1978. The Potamogetonaceae in the Southeastern of United States. J. Arnold Arbor.. 59 (2): 170-191.

Heili oust, C.B., 1980. Correlation of alkalinity and the distribution of Potamogeton in New England. Rhodora, 82: 331-344.

KADONO. Y., 1982. Occurrence of aquatic macrophytes in relation to $\mathrm{pH}$, alkalinity, $\mathrm{Ca}^{+}{ }^{+}, \mathrm{Cl}^{-}$and conductivity, Jap. J. Ecol. . 32: 39-44.

LUTIIER. H., 1947. Morphologische und Systematische beobatchtungen an Wasserphanerogamen. Acta Bot. Fenn., 40: $1-28$.

Margalef Mir, R., 1981. Distribución de los macrófitos de las aguas dulces y salobres del E y NE de España y dependencia de la composición química del medio. Fundación Juan March, Serie Universitaria, 157. Madrid. 62 pp.

Mol.ina, J.A., D. Sánchez-Mata \& J. Pizarro, 1988. Cuatro Potamogeton de España distribuidos por la «Société pour I'échange des plantes vasculaires». Soc. Ech. Plantes Vasc. Eur. Bassin Médit., Bull., 23: 83-85.

Monoyer, A., 1927. Contribution a I'anatomie et l'ethologie des monnocotylees aquatiques. Sciences, 10: 1-128.

Ogden, E.C., 1943. The broad-leaved species of Potamogeton. Rhodora, 45: 57-105, 119-163, 171-214

Pearsall, W.H. \& A.H. Hamhy, 1925. The variation of leaf form in Potamogeton perfoliatus. New Phytol., 29: 112-120.

PIP, E., 1987. The ecology of Potamogeton species in central North America. Hydrobiologia. 153: 203-216.

Raunkiapr, C., 1503. Anatomical Potamogeton-studies and Potamogeton fluitans. Bot. Tidssk., 25: 253-280.

RICHARDSON, F.D., 1980. Ecology of Ruppia maritima L. in New Hamshire (U.S.A.) tidal marshes. Rhodora, 82: $403-439$

ROMEIS, B., 1928. Taschembuch der Microscopischen Technick. Munich. 523 pp.

SCUlTHORPE, C.D., 1968. The Biology of Aquatic Vascular Plants. Edward Arnold Publ., Londres. 610 pp.

SHENK, H., 1886. Vergleichende Anatomie der Submersen Gechwächse. Cassel. $64 \mathrm{pp}$.

Talavera, S., P. Garcia Murillo \& H. Smit, 1986. Sobre el género Zannichellia. Lagascalia, 14 (2): 241-271

TOMLINSON, P.B., 1982. Potamogetonaceae. In: C.R. METCALFE (Ed.). Anatomy of Monocotyledons, 7: 270-311. University Press. Londres.

VAN WIJK, R.J., 1988. Ecological studies on Potamogeton pectinatus L.I. General characteristics, biomass production and life cycles under field conditions. Aquatic Botany. 31: 211-258.

Velayos, M., S. Cirujano \& A. Marquina, 1984. Aspectos de la vegetación acuática de la provincia de Guadalajara. Anales Jard. Bot. Madrid, 41 (1): 175-184.

WIEGLEB, G., 1988. Notes on pondwceds —outlines for a monographical treatment of the genus Potamogeton L. Feddes Reprrt., 99 (7-8): 249-266.

Wiegleb, G., W. Herk \& D. Todeskino, 1989. Ten years of vegetation dynamic in two rivulets in Lower Saxony. Vegetatio, 82: 163-178. 